\title{
Prevalence and Risk Factors of Neonatal Covid-19 Infection: A Single-Centre Observational Study
}

\author{
S. Ajith ${ }^{1}$ - V. P. Reshmi ${ }^{1}$. Sabnam Nambiar ${ }^{1} \cdot$ Ashitha Naser $^{1}$ - B. Athulya ${ }^{1}$
}

Received: 27 October 2020 / Accepted: 11 January 2021 / Published online: 4 February 2021

(c) Federation of Obstetric \& Gynecological Societies of India 2021

\begin{abstract}
Background This study is to estimate the prevalence and to determine the risk factors for neonatal Covid-19 infection Methods Retrospective analysis of all deliveries in Covid-19-infected mothers in a tertiary care centre in North Kerala from 15 April 2020 to 15 October 2020

Results Of the 350 Covid-19-positive pregnancies 223 delivered, two were intrauterine foetal demises. In total, 32 out of 221 newborns were Covid-19-positive (14.47\%). The risk was more in vaginal delivery group (17.39\%) compared to caesarean group (13.16\%). The breastfeeding and rooming-in group (18.79\%) had more infection than those babies who were not breastfed and separated from mother (1.78\%).14 out of $86(16.28 \%)$ babies delivered within 7 days of mothers turning negative became positive compared to 2 out of 23 (8.7\%) babies delivered between 7 and 14 days of negative result (Odds ratio of 2.04). None of the babies delivered 14 days after negative result has become positive.

Conclusions The present study shows that neonatal Covid-19 infection is not rare. The risk is greater in vaginal delivery group and those babies who are breastfed and allowed to stay with mothers. Delaying delivery more than 7 days after mother becoming negative protects the newborn from getting infection
\end{abstract}

Keywords Neonatal Covid-19 infection $\cdot$ Perinatal transmission

\section{Introduction}

Covid-19 pandemic has created lots of stress in pregnant women especially the concern about transmission to babies. Since it is a new disease, obstetricians are at a loss to give evidence-based answers to this question. Caesarean section, avoiding breastfeeding and mother baby separation were suggested initially to prevent perinatal transmission. Initial reports from China ruled out possibility of vertical

Dr Ajith S MD, DGO, DipNB, MRCOG, FRCOG is anAdditional Professor \& HOD, Department of OBGYN, Government Medical College Kannur, Pariyaram,Kerala; Dr Reshmi VP MS, MRCOG is anAssociate Professor, Department of OBGYN, GMC Kannur, Kerala; Dr Sabnam Nambiar MBBS, DipNB is an Associate Professor, Department of OBGYN, GMC Kannur, Kerala; Dr Ashitha Naser MBBS is aJunior Resident, Department of OBGYN, GMC Kannur, Kerala; Dr Athulya B MBBS is aJunior Resident, Department of OBGYN, GMC Kannur, Kerala.

S. Ajith

drajiths@hotmail.com

1 Department of OBGYN, Government Medical College Kannur, Pariyaram, Kerala, India transmission [1]. The report from UK of detection of IgM antibodies in cord blood of two babies suggested that vertical transmission is probable [2]. There are other papers on positive RTPCR of nasopharyngeal swab of newborns of Covid-19-infected mothers [3, 4]. However, in many of these studies, data on breastfeeding and baby separation are missing.

\section{Methods}

This is a retrospective analysis of all deliveries of Covid19-infected mothers in a tertiary care centre in north Kerala from April 15, 2020 to October 15, 2020. The objective of the study is to estimate the prevalence of neonatal Covid-19 infection and to determine the risk factors for the same. The hospital is a referral centre for two northern districts of Kerala. The state policy is to screen for Covid-19, all pregnant ladies, at time of hospital admission for delivery. The test is also done in all pregnant ladies with Covid symptoms, contact or travel abroad or other states. The usual test is rapid antigen test and if negative RTPCR of nasopharyngeal swab. 
The test is repeated after 10 days of positive result. If the test is still positive, it is repeated on alternate days till negative. All newborns delivered to Covid-positive mothers (even if they turned negative at the time of delivery) are tested for Covid-19 within $24 \mathrm{~h}$ of delivery by RTPCR (Reverse Transcription Polymerase Chain Reaction) of nasopharyngeal swab. The positive babies are followed up for any symptoms, such as fever, gastrointestinal symptoms, hypoxia and cough and for swab negative status. The following factors are studied to determine relation to perinatal transmission-route of delivery (vaginal or caesarean), mother's Covid status at delivery (including interval between negative status and delivery if mother turned negative), severity of infection (severe infection needing intensive care unit admission or non-severe infections), prematurity (less than 37 completed weeks or term), breastfeeding and rooming-in. The mothers Covid status at delivery is noted as positive or negative and if negative documentation is done whether delivery occurred within 1 week, 2 weeks or more than two weeks of delivery. All these data were collected as part of an ongoing prospective study and were entered in the data sheet keeping confidentiality. The frequency and percentage of all these factors were used to describe data. The data were analysed by means of Open Epi version 3.01 software. Pearson Chi-square test was applied, and $P$ value of $<0.05$ was considered statistically significant.

\section{Results}

In this period, we had managed 350 Covid-19-positive pregnant ladies, out of whom 223 delivered. There were 152 caesarean Sect. (68.16\%) and 71 vaginal deliveries (31.84\%). A total of 18 babies delivered preterm $(8.1 \%)$ - 16 caesarean $(88.89 \%)$ and two vaginal deliveries $(11.11 \%)$. There were two cases of intrauterine foetal demise-in these cases, we could not blame Covid infection as the cause of intrauterine demise, as they were found to be macerated babies, patients were asymptomatic, and diagnosis made on screening at hospital admission after diagnosis of intrauterine foetal demise. There were nine miscarriages. The main indications for primary caesarean section were foetal distress $(42 \%)$, cephalon pelvic disproportion (16\%), foetal growth restriction (12\%), abruption (5\%), failed induction (5\%), severe preeclampsia (4\%) and abnormal presentation (4\%).

Out of 221 babies 32 babies turned positive (14.47\%). A total of 20 babies out of 152 caesarean deliveries turned positive and 12 babies out of 69 vaginal deliveries turned positive (Table 1

The risk of getting neonatal Covid in vaginal delivery group was $17.39 \%$, and the risk in caesarean group was $13.16 \%$ (vaginal delivery has an Odds Ratio of 1.389). The nasopharyngeal swab of all newborns were taken for Covid19 testing by RTPCR within $24 \mathrm{~h}$ of birth, even if the mothers turned negative.

The risk of baby being positive if delivered while mother is positive was $14.29 \%$ and when mother turned negative was $14.68 \%$ (Odds ratio 0.9688 ) that means there is not much difference between two groups. One suggestion was delaying delivery more than 1 week after test turning negative-it could protect the babies from infection. A total of 14 out of $86(16.28 \%)$ babies delivered within 1 week of mother turning negative became positive, but only two out of 23(8.7\%) babies delivered between seven and 14 days after negative result became positive. (Odds ratio 2.04) None of the babies delivered 14 days after negative result turned positive (Table 2).

Next parameter assessed is whether the severity of maternal infection increases the risk of perinatal transmission. In our study, only two mothers had severe infection, needing
Table 1 Risk factors for neonatal covid-19 infection

\begin{tabular}{lll}
\hline Variables & $\begin{array}{l}\text { Neonatal Covid positive } \\
\text { by RTPCR } n=32\end{array}$ & $\begin{array}{l}\text { Neonatal Covid } \\
\text { negative by RTPCR } \\
n=189\end{array}$ \\
\hline Caesarean section & 20 & 132 \\
Vaginal delivery & 12 & 57 \\
Mother Covid positive at time of delivery & 16 & 96 \\
Mother turned Covid-negative at time of delivery & 16 & 93 \\
Mother had severe Covid-19 & 0 & 2 \\
Mother had non-severe Covid-19 & 32 & 187 \\
Preterm birth & 2 & 13 \\
Term birth & 30 & 176 \\
Breastfeeding & 31 & 134 \\
No breastfeeding & 1 & 55 \\
Rooming-in & 31 & 134 \\
No rooming-in & 1 & 55 \\
\hline
\end{tabular}


Table 2 Risk of neonatal covid-19 infection if delivery occurs after mother becoming test negative

\begin{tabular}{lll}
\hline & $\begin{array}{l}\text { Neonatal Covid posi- } \\
\text { tive } n=16\end{array}$ & $\begin{array}{l}\text { Neonatal Covid } \\
\text { negative } N=93\end{array}$ \\
\hline $\begin{array}{l}\text { Mothers delivered }<7 \\
\text { days after turned Covid- } \\
\text { negative }\end{array}$ & 14 & 72 \\
$\begin{array}{l}\text { Mothers delivered } \\
7-14 \text { days after turned } \\
\text { Covid-negative }\end{array}$ & 2 & 21 \\
\hline
\end{tabular}

ICU admission. One mother needed invasive ventilation. Nevertheless, both babies were negative. The incidence of preterm delivery was $8.1 \%$. Risk of getting neonatal Covid19 infection in preterm babies was $13.33 \%$, compared to $14.56 \%$ in term babies (Odds ratio of 0.9026), not much difference. The hospital policy is to allow breastfeeding and rooming-in after discussing with parents. Those opting for breastfeeding and rooming-in are advised regarding hand hygiene, use of mask and keeping the baby at a proper distance in the room. Those opting not to breastfeed are advised formula feed by a Covid-negative person. Risk of neonatal Covid-19 infection with breastfeeding and rooming-in was $18.79 \%$ compared to $1.78 \%$ in the nonexposed group (Odds ratio of 12.72).

\section{Discussion}

The initial reports of Covid-19 infection in pregnant women did not support the possibility of vertical transmission [1]. A case report of neonatal Covid-19 infection was reported from China in March 2020 [3]. There were two reports of possible vertical transmission in the form of elevated Covid19 IgM antibodies in three newborns although repeated nasopharyngeal samples from infants were negative. A systematic review and meta-analysis showed a relatively low rate of neonatal infection highlighting a low probability of vertical transmission [5]. Most babies tested positive for Covid-19 were asymptomatic, even though there were few reports of severe Covid-19 illness in the newborn.

As per CDC the risk of newborn getting Covid-19 from its mother is low, especially if she uses appropriate precautions before and during care of the newborn, such as wearing a mask and practicing hand hygiene. Keeping the baby with the mother ("rooming-in") has the benefit of facilitating breastfeeding and maternal-newborn bonding. The precautions recommended are washing the hand with soap and water for at least $20 \mathrm{~s}$ before holding or caring the baby, keeping the baby more than six feet away from the mother and wearing a mask. The current evidence also support breastfeeding. The breast milk provides protection against many illnesses and is the best source of nutrition for most babies [6].

In our study Caesarean section rate was $68.1 \%$ which is comparable to other studies. UKOSS study showed a caesarean rate of 59\% [7], and a systematic review published in September 2020 issue of European Journal of Obstetrics and Gynecology and Reproductive Biology showed a caesarean section rate of $72 \%$ [8]. The same studies showed a higher preterm delivery rate of $27 \%$ and $23 \%$, respectively. The living systematic review and meta-analysis published in September issue of British Medical Journal also concluded a higher preterm delivery (17\%) in Covid-19-infected pregnant ladies [9]. But our study showed a preterm delivery of only $8.1 \%$.

A systematic review [4] published in October issue of BJOJ, based on 49 studies including 655 pregnant women and 666 neonates shows that neonatal Covid-19 infection is uncommon, almost never symptomatic and the rate of infection is no greater when the baby is born vaginally, breast fed or allowed contact with the mother. As per that study, only $4 \%$ of neonates were tested positive. Of the babies born vaginally $2.7 \%$ tested positive compared with $5.3 \%$ born by Caesarean. Information on feeding and baby separation was often missing, but of reported breast-fed babies 7/148 (4.7\%) tested positive compared with 3/56 (5.3\%) for reported formula-fed babies. Of babies reported as nursed with their mother $4 / 107$ (3.7\%) tested positive compared with 6/46 (13\%) for those who were reported as isolated. Our study shows that 32 out of 221 babies were tested positive (14.47\%). The neonates tested positive was more in vaginal delivery group (17.39\%) compared to caesarean group (13.16\%) giving an odds ratio of 1.389 . We have collected proper information regarding breastfeeding and rooming-in in all these cases. The mothers were properly counselled regarding breastfeeding and rooming-in, and the parental decision was respected. Of breastfed and rooming-in babies $18.79 \%(31 / 165)$ tested positive compared to $1.78 \%(1 / 56)$ who were not breastfed and separated from mother giving an odds ratio of 12.72 .

The induction of labour is generally postponed in Covidpositive pregnant ladies if there is no definite indication [2]. We analysed if this has any effect on neonatal infection. The risk of neonatal infection when delivered while mother is positive was $14.29 \%$ compared to $14.68 \%$ when delivered after mother becoming negative. There was no significant difference between the groups (Odds ratio 0.9688). Then, we analysed whether delaying delivery more than 7 days have any effect on test positivity. In total, 14 out of $86(16.28 \%)$ babies delivered within 7 days of mothers turning negative became positive compared to 2 out of $23(8.7 \%)$ babies delivered between 7 and 14 days of negative result (Odds ratio of 2.04). None of the babies delivered 14 days after 
negative result has become positive. Hence, delaying delivery by more than seven days after mother turning negative has a protective effect on baby getting infection.

In our study, only two mothers had severe infection, but babies of both mothers were negative. Out of 32 positive babies, only two were preterm. The risk of getting neonatal infection in preterm babies was $13.33 \%$ compared to $14.56 \%$ in term babies (Odds ratio 0.9026 ). The positive newborns were asymptomatic, only 2 babies were admitted in neonatal ICU for suspected mild sepsis and none of the babies needed prolonged hospital stay.

\section{Conclusion}

The present study shows that neonatal Covid-19 infection is not rare. The risk is more in vaginal delivery group and those babies who are breastfed and allowed to stay with mothers. Delaying delivery by more than 7 days after mother turning negative protects the newborn from infection. The neonatal infections are mostly asymptomatic.

The strength of the study is that it is conducted in a single centre and data are properly collected. The chief weakness is small sample size.

\section{Compliance with ethical standards}

Conflict of interest The authors that they have declare no conflict of interest.

Ethical statement Approval obtained from Institutional Ethical Committee.

\section{Reference}

1. Chen H, Guo J, Wang C, et al. Clinical characteristics and intrauterine vertical transmission potential of COVID 19 infection in nine pregnant women: a retrospective review of medical records. Lancet. 2020;395(10226):809-15.

2. RCOG. Corona virus (Covid-19) infection in pregnancy. Information for healthcare professionals: Version 6: published Friday 3 April 2020.
3. Dong L, Tian J, He S, et al. Possible vertical transmission of SARS-CoV-2 from an infected mother to her newborn. JAMA. 2020;323(18):1846-8.

4. Walker KF, Donoghue KO, Grace N, et al. Maternal transmission of SARS-COV 2 to the neonate, and possible routes of such transmission: a systematic review and critical analysis. BJOG. 2020;127(11):1324-36.

5. Capobianco Giampiero, Saderi Laura, Aliberti Stefano, et al. Covid-19 in pregnant women: a systematic review and metaanalysis. J Obstet Gynecol Reprod Biol. 2020;252:543-58.

6. CDC. Information on pregnancy, Breast feeding and Caring for Newborns during the COVID-19 Pandemic updated October 23, 2020.

7. RCOG. Corona virus (Covid-19) infection in pregnancy. Information for healthcare professionals: Version 12: published Wednesday 14 October 2020.

8. Dubey Pallavi, Reddy Sireesha Y, Manuel Sharon, et al. Maternal and neonatal characteristics and outcomes among COVID-19 infected women: an updated systematic review and meta-analysis. Eur J Obstet Gynecol Reprod Biol. 2020;252:490-501.

9. Allotey John, Stallings Elena, Bonet Mercedes, et al. Clinical manifestations, risk factors, and maternal and perinatal outcomes of corona disease 2019 in pregnancy: living systematic review and meta-analysis. BMJ. 2020;370:3320.

Publisher's Note Springer Nature remains neutral with regard to jurisdictional claims in published maps and institutional affiliations.

\section{About the Author}

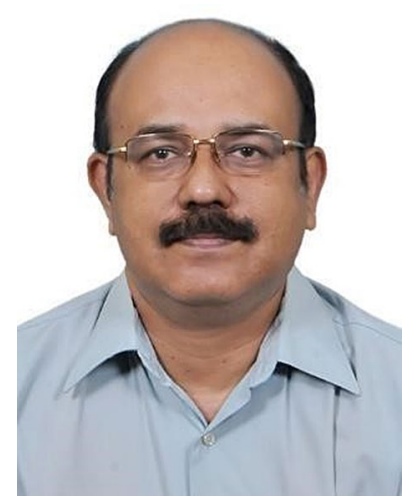

S. Ajith Dr Ajith $\mathrm{S}$ is the Head of the department of Obstetrics and Gynaecology in Government Medical College Kannur. He is the state coordinator of maternal near miss audit of Kerala Federation of Obstetricians and Gynaecologists. He is leading a multicentric trial on foetal and maternal outcome in Covid-19 affected pregnancies in government medical colleges of Kerala. 